\title{
IL-17A polymorphism (rs2275913) and levels are associated with preeclampsia pathogenesis in Chinese patients
}

\author{
Xiao Lang ${ }^{1,2 \dagger}$, Wei Liu, ${ }^{1,2 \dagger}$, Yanyan Hou ${ }^{1,2}$, Wenxia Zhao ${ }^{3}$, Xingyu Yang ${ }^{1,4}$, Lan Chen ${ }^{1,2}$, Qi Yan ${ }^{3}$ \\ and Weiwei Cheng ${ }^{1,2^{*}}$ (D)
}

\begin{abstract}
Background: Preeclampsia (PE) is a pregnancy-related condition that affects both the infant and the mother. Although the role of various inflammatory molecules in PE has been demonstrated, the importance of pro-inflammatory molecules such as IL-17A, IL-23 is not well understood. In the present investigation, a potential association of common genetic variants in the IL-17A and IL-23A genes with PE was investigated.

Methods: 115 PE clinically diagnosed patients who registered to the International Peace Maternity and Child Health Hospital were enrolled in this research. One hundred two pregnant women and 147 healthy Chinese women were also included. ELISA was used to measure IL-17A and IL-23 serum levels in all enrolled subjects. Common genetic polymorphisms in IL-17A (rs 2,275,913, rs1974226, and rs1974226), IL-23A (rs11171806), and IL-12B (rs3212227) were genotyped using the PCR-RFLP or TaqMan probe-based method.

Results: Elevated serum IL-17A levels were found in PE patients compared to pregnant $(P<0.0001)$ and healthy women $(P<0.0001)$. However, IL-23 levels were comparable across various clinical groups. In addition, heterozygous (GA) and minor allele (A) for IL-17A (rs2275913) and IL-23A (rs11171806) were more prevalent in PE patients compared to pregnant women indicating an important role in the predisposition to PE growth. Interestingly, IL-17A ( 2 2,275,913) mutants were associated with elevated IL-17A levels relative to wild type (GG).
\end{abstract}

Conclusions: IL-17A (rs2275913) variants are associated with higher serum levels of cytokine, and predisposed PE development.

Keywords: Preeclampsia, IL-17A, IL-23A, Gene polymorphism, Serum levels

\section{Background}

Preeclampsia (PE) is a complication associated with pregnancy and is characterised by high blood pressure and dysfunction of different organ systems. PE syndrome has a deleterious effect on both the mother and the

\footnotetext{
*Correspondence: wwcheng29@shsmu.edu.cn

${ }^{\dagger}$ Xiao Lang and Wei Liu contributed equally to this work.

${ }^{1}$ The International Peace Maternity and Child Health Hospital, School

of Medicine, Shanghai Jiao Tong University, 910 Hengshan Road,

Shanghai 200030, China

Full list of author information is available at the end of the article
}

developing foetus. Although the exact cause and pathogenesis of this disease are not known, it is suspected that the disease is guided by a variety of factors caused by placental pressure-induced trophoblasts, which promote overwhelming maternal inflammatory response [1]. It is estimated that about 10 million people are developing PE per year worldwide [2]. In addition, approximately 76,000 pregnant women die each year as a result of $\mathrm{PE}$ and approximately 500,000 children die annually as a result of PE [3]. Epidemiological studies of PE in the Chinese population are very limited: a retrospective analysis 
in three separate hospitals in China found around 2.35\% $\mathrm{PE}$ in a total of 67,746 pregnant women and PE was most prevalent in nulliparity subjects $(81.5 \%)$ [4]. There is a wide repertoire of immunological modulators and signalling pathways that lead to the initiation and progression of PE. Previous evidences indicate that cytokines play a critical role in controlling various stages of pregnancy among different immune molecules $[5,6]$. Th1: Th2 dichotomy during pregnancy indicated that Th2 mediated immunity is involved in the maintenance of normal occurrences during pregnancy, while Th1 form of immune response is associated with pregnancy-related problems such as miscarriage [7], premature delivery [8], rupture of foetal membranes prior to the start of labour pain [9]. Previous studies have suggested a difference in preeclamptic placenta levels of cytokines, leading to complicated conditions such as delayed intrauterine development and preterm delivery [10]. Cytokine IL-17 was derived from Th17 lymphocytes recently discovered as a subset of CD4+T lymphocytes [11]. Several studies indicated a higher percentage of the population of Th17 cells in complicated pregnancy cases, such as abortion, preterm birth or PE [12, 13, 14].

Interlukin-23 (IL-23) is a proinflammatory cytokine that is responsible for Th-17 cell discrimination, spread, and survival [15]. In the pathogenesis of PE, upregulation of the Th-17 cell-mediated immune response has been demonstrated [16]. IL-23 is a cytokine heterodimer consisting of subunits IL-12B and IL-23A. There have been earlier reports of differential levels of IL-23 in preeclamptic patients [12]. Based on the importance of IL-23 in controlling inflammation, we hypothesised that IL-23 cytokine could be linked to the clinical condition of PE patients in a Chinese cohort,.

Functional single nucleotide polymorphisms (SNPs) have been associated with the variance of serum cytokine levels in subjects. Although several SNPs are reported in the IL-17A gene, common polymorphisms like rs2275913 ( $-197 \mathrm{G}>\mathrm{A}$ ), rs1974226 (3'UTR C > T), and rs3748067 $(1249 \mathrm{C}>\mathrm{T})$ are widely investigated on genetic association studies. Similarly, variants of IL-23A (rs11171806: $\mathrm{A}>\mathrm{G}$, exon 106Ser $>$ Ser) and IL-12B (rs3212227 A $>$ C 3 'UTR) also investigated in various reports and their association with susceptibility to a wide range of diseases has already been established. Investigations to decipher genetic association of IL-17A, IL-23, and IL-12B common variants with $\mathrm{PE}$ is limited. Earlier reports failed to demonstrate possible association of IL-17A polymorphism (rs2275913) with susceptibility to PE in iraninan and Chinese women $[17,18]$. However, other functional SNPs in the IL-17A gene has not been explored. Further, IL-12B (rs3212227) polymorphism was also not linked with PE predisposition in Han Chinese [19]. To best of our knowledge, association of IL-23A genetic variants with PE predisposition has not been studied.

Simultaneous investigation into the relationship between IL-17A, IL-23A and IL12B common genetic polymorphisms with predisposition to $\mathrm{PE}$ and their respective serum levels with pathogenesis of PE is missing in the Chinese population. In this study, a hospital-based case control investigation was conducted to decipher the combination of IL-17A, IL-23A and IL12B genetic variants and their functional significance in $\mathrm{PE}$ pathophysiology.

\section{Methods}

\section{Study subjects}

The present study was performed between a period from March 2017 to December 2019 at the International Peace Maternity and Child Health Hospital. The study protocol was approved by the Institutional Review Board of Shanghai Jiao Tong University and the informed signed consent was obtained from all participants. The present investigation was a hospital based case control study. The first category included 120 patients with PE. The primary inclusion criteria for these patients was third-trimester pregnancy complicated with $\mathrm{PE}$, blood pressure $>140 / 90$ with proteinuria $>300 \mathrm{mg}$ in $24 \mathrm{~h}$ (According to ACOG) [20]. The second category included 120 women in thirdtrimester pregnancy without PE. The third category included 150 healthy non-pregnant women and was used as controls. Patients with microvascular complications, co-existing autoimmune, chronic/acute inflammatory diseases, multiple gestations, diabetes mellitus, sickle cell disease, and HIV infection were excluded from the present investigation. Clinical characteristics data was gathered from hospital records.

\section{Collection of serum}

Three milliliters of blood samples (without anticoagulant) collected intravenously from pregnant women and nonpregnant controls before starting therapy. The serum was separated from each sample by centrifuging blood at $950 \mathrm{~g}$ for $5 \mathrm{~min}$. The supernatant was collected and retained at $-80^{\circ} \mathrm{C}$ for future quantification of cytokines.

\section{Cytokines (IL-17A and IL-23) quantification}

Serum levels IL-17A or IL-23 were quantified by enzymelinked immunosorbent assays (ELISA) using the predesigned kit as per the manufacturer's instructions (R\&D Systems, Inc., USA) in all subjects enrolled for the present investigation. 


\section{Genomic DNA isolation}

For genomic DNA isolation, 200ul of whole blood was used. SIGMA mini Genomic DNA extraction kit was used for isolation of whole genomic DNA.

\section{Genotyping of IL-17A, IL-12B and IL-23A polymorphisms}

A polymerase chain reaction followed by restriction fragment length polymorphism (PCR-RFLP) technique was employed for the genotyping of IL-12B (rs3212227), as described by an earlier report [21]. Briefly, two primers (forward: GATATCTTTGCTGTATTTGTATAGTT and reverse: AATATTTAAATAGCATGAAGGC) were used for amplification of a $118 \mathrm{bp}$ gene fragment flanking the polymorphic site. The thermal cycler conditions were as follows: early denaturation at $95^{\circ} \mathrm{C}$ for $5 \mathrm{~min}$ followed by 35 cycles of $95^{\circ} \mathrm{C}$ for $40 \mathrm{~s}, 55^{\circ} \mathrm{C}$ for $35 \mathrm{~s}$, and at $72^{\circ} \mathrm{C}$ for $25 \mathrm{~s}$. The final extension reaction was performed at $72{ }^{\circ} \mathrm{C}$ for $10 \mathrm{~min}$. The amplicon was digested with TaqI restriction enzyme and fragments were analysed for rs3212227 polymorphism. Amplicon with mutant allele has a restriction site for TaqI, thus produces two fragments $(92 b p+26 b p)$; on the other hand, for wildtype allele, the amplicon remained undigested (118bp). Similarly, the PCR-RFLP technique was also used for genotyping of IL-17A (rs2275913, rs1974226, and rs3748067) polymorphisms, as described in an earlier report [22]. Following primers were used for amplification of IL-17A gene bordering SNPs sites and yield different amplicons (rs2275913: forward- GCTCAGCTTCTAACAAGT AAG, reverse- AAGAGCATCGCACGTTAGTG, amplicon size-338bp; rs1974226: forward- AAAGGAGCT GATGGGGCAGTA, reverse- GGTCTTTCAAGAAGC AGGGAG, amplicon size-211; rs3748067: forward- GGG CTGAACTTTTCTCATACTTAGA, reverse- GAGACA TTGTCTTCAGACTACAATG, amplicon size-212bp). The annealing temperature for genotyping of IL-17A polymorphisms was fixed at $58^{\circ} \mathrm{C}$, and other conditions were like those of IL-12B. Different restriction enzymes were used (rs2275913: EarI, rs1974226: RsaI and rs3748067: EcoRI) for digestion of the amplicon and based on differential digested DNA fragments, genotypes of subjects were determined as follows ( $r 2275913: \mathrm{A}=259+79 \mathrm{bp}$, $\mathrm{G}=338 \mathrm{bp} ; \mathrm{rs} 1974226: \mathrm{A}=221 \mathrm{bp}, \mathrm{G}=191+20 \mathrm{bp}$ and rs3748067:G=212bp, $A=198+24 \mathrm{bp}) . \quad$ As described earlier [23], the IL-23A (rs11171806) gene polymorphism was genotyped by the TaqMan PCR assay by using a TaqMan probe. In brief, predesigned SNP genotyping assays kit were procured from Thermo Fisher Scientific (rs11171806: C_25985467_10, VIC/FAM- TTTTTT ATGAGAAGCTGCTAGGATC[A/G]GATATTTTCACA GGGGAGCCTTCTC) and the typing was performed in Applied Biosystems Realtime PCR system $(7900 \mathrm{HT})$ as directed by the manufacturer.

\section{Statistical analysis}

Graphpad prism v8.2 was employed for all statistical analyses. Serum levels of IL-17A and IL-23 in different clinical groups were compared by one-way analysis of variance (ANOVA) and the mean cytokines levels of all groups were compared with those of every other clinical categories or genotypes by Tukey's post-test. Genotype and allele frequencies were calculated by manual counting. Genotypes distribution of all studied SNPs were tested for Hardy Weinberg equilibrium with in house developed Microsoft excel file. Distribution of genotypes and alleles were compared with Fisher exact test in different clinical categories, odds ratio and 95\% confidence interval was calculated. A $P$ value of less than 0.05 was taken as significant.

\section{Results}

\section{Baseline characteristics of patients and controls}

A total of 120 cases of PE and 120 pregnant women were included in the study. Both plasma cytokines and genetic polymorphisms was successfully analysd in 115 PE patients and 102 pregnant women. Out of 150 healthy Chinese women included in the present investigation, IL-17, IL-23 and IL-12B polymorphisms and plasma cytokines levels were efficiently quantified in 147 subjects. Thus based on availability of data for both genotypes and levels of cytokines, a total of 364 females were considered in the present study comprising $115 \mathrm{PE}$ patients, 102 pregnant women, and 147 non-pregnant women. Different baseline characteristics were compared among clinical categories (Table 1). A significant difference was observed in different parameters while comparing PE cases and pregnant women, such as duration of gestation (days), body mass index $(\mathrm{Kg} / \mathrm{m} 2)$, vaginal delivery (\%), caesarian section (\%), fetal birth weight (gram), systolic/diastolic blood pressure $(\mathrm{mmHg}), \mathrm{WBC}$ count $\left(\times 10^{9} / \mathrm{L}\right)$, levels of urea and uric acid $(\mathrm{mg} / \mathrm{dL})$. However, percentage of pre-eclamptic patients with primiparas were not significantly altered in comparison to healthy pregnant women.

\section{PE patients displayed higher serum IL-17A compared to controls}

The serum levels of IL-17A and IL-23 were quantified by ELISA. As shown in Fig. 1a, the mean level of IL-17A was $59.1 \pm 0.93 \mathrm{pg} / \mathrm{ml}$ in healthy women without pregnancy, whereas healthy pregnant women and subjects with PE had $61.13 \pm 1.43 \mathrm{pg} / \mathrm{ml}$ and $746.7 \pm 17.16 \mathrm{pg} /$ $\mathrm{ml}$ of IL-17A level, respectively. Although a comparable level in IL-17A was observed between healthy pregnant and non-pregnant women, subjects with PE demonstrated a noticeably higher cytokine level as compared to two other study groups, suggesting an essential 
Table 1 Baseline characteristics of study subjects

\begin{tabular}{llll}
\hline Parameters & Subjects with PE $(\boldsymbol{n = 1 1 5})$ & Pregnant women $(\boldsymbol{n = 1 0 2})$ & $\begin{array}{c}\text { Non-pregnant } \\
\text { women } \\
(\boldsymbol{n}=\mathbf{1 4 7})\end{array}$ \\
\hline Age (years) & & & $29 \pm 4$ \\
Primiparas (\%) & $31 \pm 5$ & $33 \pm 6$ & $\mathrm{NA}$ \\
Duration of gestation (days) & 59.3 & 54.9 & $\mathrm{NA}$ \\
BMl at blood draw (Kg/m2) & $249 \pm 14^{*}$ & $269 \pm 19$ & $21.1 \pm 3.8$ \\
Vaginal delivery (\%) & $30.1 \pm 5.2^{*} \#$ & $26.2 \pm 4.1 \#$ & $\mathrm{NA}$ \\
Caesarian section (\%) & $12.3^{*}$ & 56.6 & $\mathrm{NA}$ \\
Fetal birth weight (grams) & $87.7^{*}$ & 43.4 & $\mathrm{NA}$ \\
Systolic blood pressure (mmHg) & $2651^{*}$ & 3216 & $113 \pm 11$ \\
Diastolic blood pressure $(\mathrm{mmHg})$ & $149 \pm 13^{*} \#$ & $109 \pm 09$ & $80 \pm 10$ \\
White blood cell $\left(\times 10^{9} / \mathrm{L}\right)$ & $89 \pm 11^{*} \#$ & $75 \pm 09$ & $8.8 \pm 2.9$ \\
Uric acid (mg/dL) & $10.1 \pm 3.5^{*} \#$ & $9.6 \pm 3.2 \#$ & $3.9 \pm 1.9$ \\
Urea (mg/dL) & $7.1 \pm 0.9^{*} \#$ & $4.2 \pm 1.3$ & $17.6 \pm 4.4$ \\
\hline
\end{tabular}

Data are presented as either mean \pm S.D. or in percentage (\%)

NA Not applicable

${ }^{*} P<0.05$ - Subjects with PE versus healthy pregnant women; $P<0.05$ - Subjects with PE versus healthy non pregnant women
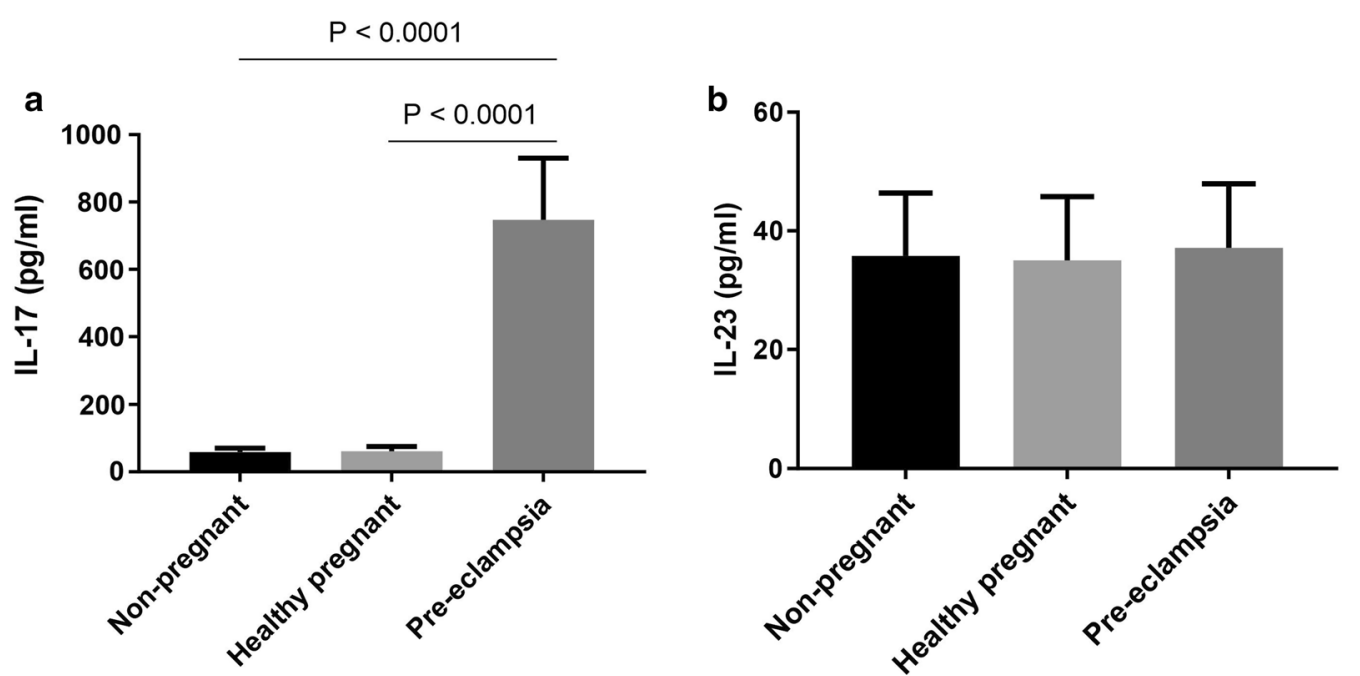

Fig. 1 Serum cytokines levels in different categories of enrolled subjects. Serum level IL-17 (a) and IL-23 (b) in subjects with PE ( $n=115)$, healthy pregnant women $(n=102)$ and healthy non pregnant controls $(n=147)$. Data represent mean $\mathrm{pg} / \mathrm{ml} \pm \mathrm{SE}$ and were analyzed with one way ANOVA for comparison. $P<0.05$ was considered statistically significant

role of this molecule in promoting pathogenesis during PE. Further, to assesses the importance of IL-23 in regulating the pathologic condition of $\mathrm{PE}$, the titer of the cytokine was measured in sera and the results are shown in Fig. 1b. The results showed a relatively similar level of this cytokine in all the three groups.
Distribution of IL-17A, IL-23A and IL-12B polymorphisms in healthy non-pregnant women

The genotype and allele frequency of IL-17A (rs2275913, rs1974226, and rs3748067), IL-23A (rs11171806) and IL12B (rs3212227) polymorphisms in healthy female and the distribution of genotypes for all SNPs are in HardyWeinberg equilibrium (rs2275913: $X^{2}=2.58, P=0.10$; rs1974226: $X^{2}=2.83, \quad P=0.08 ; \quad$ rs3748067: $X^{2}=0.17$, 
$P=0.67 ; \quad$ rs $11171806: X^{2}=1.93, \quad P=0.16 ; \quad$ rs3212227: $\left.X^{2}=0.01, P=0.90\right)($ Table 2).

\section{IL-17A (rs2275913) and IL-23A (rs11171806)} polymorphisms are associated with predisposition to $\mathrm{PE}$ As shown in Table 2. Heterozygous (GA) and minor allele (A) of IL-17A (rs2275913) polymorphism were significantly more prevalent in PE patients compared to the pregnant women (GA: $P=0.007, \mathrm{OR}=2.40$; A: $P=0.02$, OR $=1.54$ ). No significant genetic association was observed in the distribution of other IL-17A polymorphisms (rs1974226 and rs3748067) and IL-12B (rs3212227) in PE patients in comparison to the pregnant women. Interestingly, when we analyzed association of IL-23A polymorphism (rs11171806) with predisposition to development of PE, a significant link of heterozygous variants and minor allele were noticed with susceptibility to PE development (GA: $P=0.008, \mathrm{OR}=3.24$, A: $P=0.004$, OR $=3.27$ ).

\section{Genotype-phenotype association of IL-17A and IL-23A polymorphisms}

AA and GA genotypes of rs2275913 polymorphisms displayed significantly higher serum IL-17A compared to wildtype (GG) (Fig. 2a). Serum IL-17A levels were comparable among different genotypes of rs1974226 and rs3748067 polymorphisms (data not shown). Furthermore, variants of IL-23A (rs11171806) also failed to demonstrate any functional relevance on serum levels of IL-23A (Fig. 2b).

Table 2 Distribution of IL-23A, IL-12B and IL-17A gene polymorphisms in healthy controls, pregnant woman and preeclampsia patients

\begin{tabular}{|c|c|c|c|c|}
\hline Genotype/Allele & $\mathrm{HW}(n=147)$ & $\mathrm{PW}(n=102)$ & $\mathrm{PE}(n=115)$ & PW vs $\mathrm{PE}$ ( $P$ value, $\mathrm{OR}, 95 \% \mathrm{Cl})$ \\
\hline \multicolumn{5}{|l|}{ IL-23A (rs11171806) } \\
\hline GG & $135(92)$ & $95(93)$ & $92(80)$ & 1, ref \\
\hline GA & $11(7)$ & $7(7)$ & $22(19)$ & $0.008,3.24,1.31$ to 8.53 \\
\hline$A A$ & $1(1)$ & 0 & $1(1)$ & - \\
\hline G & $281(96)$ & $197(97)$ & $206(90)$ & 1, ref \\
\hline A & $13(4)$ & $7(3)$ & $24(10)$ & $0.004,3.27,1.44$ to 8.18 \\
\hline \multicolumn{5}{|l|}{ IL-12B (rs3212227) } \\
\hline AA & $125(85)$ & $89(87)$ & $93(81)$ & 1 , ref \\
\hline$A C$ & $21(14)$ & $12(12)$ & $20(17)$ & $0.25,1.59,0.75$ to 3.47 \\
\hline CC & $1(1)$ & $1(1)$ & $2(2)$ & $1,1.91,0.21$ to 28.01 \\
\hline A & $271(92)$ & $190(93)$ & $206(90)$ & 1 , ref \\
\hline C & $23(8)$ & $14(7)$ & $24(10)$ & \\
\hline \multicolumn{5}{|l|}{ IL-17A (rs2275913) } \\
\hline GG & $54(37)$ & $39(38)$ & $24(21)$ & 1 , ref \\
\hline GA & $62(42)$ & $44(43)$ & $65(56)$ & $0.007,2.40,1.29$ to 4.47 \\
\hline AA & $31(21)$ & $19(19)$ & $26(23)$ & $1,0.96,0.47$ to 1.96 \\
\hline G & $170(58)$ & $122(60)$ & $113(49)$ & 1 , ref \\
\hline$A$ & $124(42)$ & $82(40)$ & $117(51)$ & $0.02,1.54,1.05$ to 2.25 \\
\hline \multicolumn{5}{|l|}{ IL-17A (rs1974226) } \\
\hline $\mathrm{CC}$ & $129(88)$ & $87(85)$ & $102(89)$ & 1, ref \\
\hline$C T$ & $16(11)$ & $13(13)$ & $13(11)$ & $0.83,0.85,0.37$ to 1.94 \\
\hline $\mathrm{TT}$ & $2(1)$ & $2(2)$ & 0 & - \\
\hline$C$ & $274(93)$ & $187(92)$ & $217(94)$ & 1, ref \\
\hline $\mathrm{T}$ & $20(7)$ & $17(8)$ & $13(6)$ & $0.34,0.65,0.32$ to 1.36 \\
\hline \multicolumn{5}{|l|}{ IL-17A (rs3748067) } \\
\hline CC & $119(81)$ & $82(80)$ & $91(79)$ & 1 , ref \\
\hline $\mathrm{CT}$ & $26(18)$ & $20(20)$ & $24(21)$ & $0.86,1.08,0.56$ to 2.08 \\
\hline $\mathrm{TT}$ & $2(1)$ & 0 & 0 & - \\
\hline$C$ & $264(90)$ & $184(90)$ & $206(90)$ & 1, ref \\
\hline $\mathrm{T}$ & $30(10)$ & $20(10)$ & $24(10)$ & $0.87,1.07,0.58$ to 2.04 \\
\hline
\end{tabular}

Data are presented in number (\%)

$H W$ Healthy non-pregnant woman, PW Pregnant woman, PE Preeclampsia patients, OR Odds ratio, CI Confidence interval 

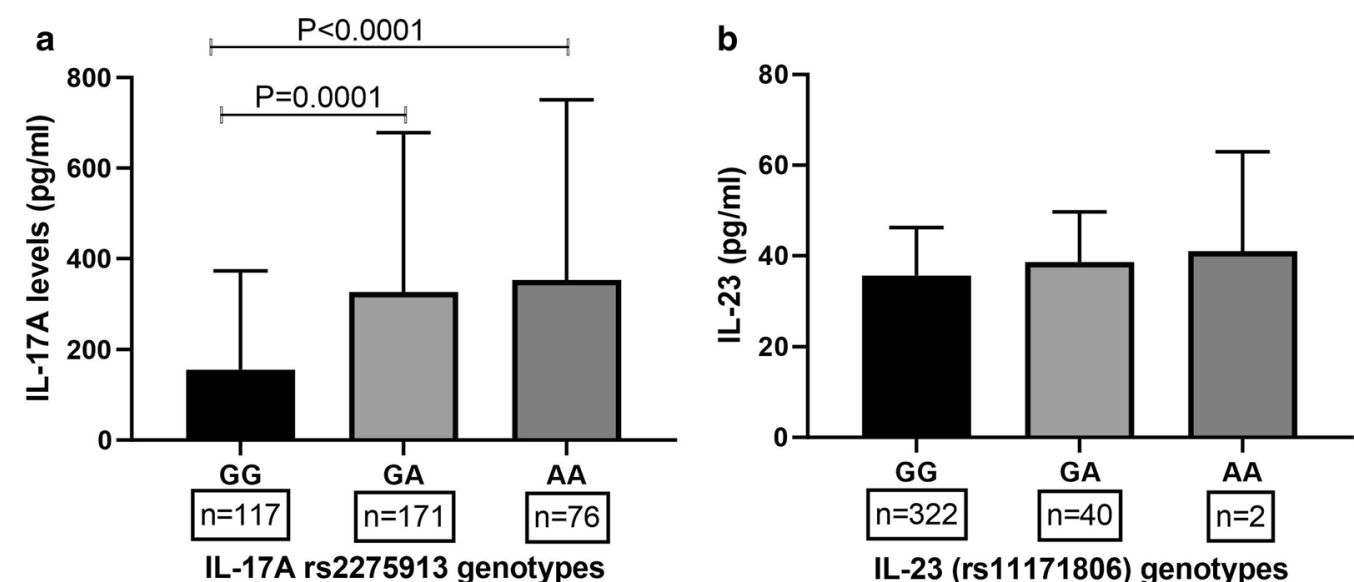

Fig. 2 Association of IL-17A (rs2275913) and IL-23 (rs11171806) polymorphism with respective serum levels. Serum levels of IL-17A were quantified by ELISA and rs2275913 polymorphism was typed by PCR-RFLP in a total of 364 subjects comprising of healthy females, pregnant woman, and subject with preeclampsia (a). Mean IL-17A levels in the different genotype of rs2275913 polymorphism were compared by ANOVA followed by Tukey's post-test. No significant association between IL-23A (rs11171806) and levels of IL-23 was observed (b). A P value of less than 0.05 was taken as significant

\section{Discussion}

The importance of proinflammatory molecules in $\mathrm{PE}$ pathogenesis has been deciphered by several studies. However, the role of IL-17 and IL-23 in PE has not been extensively investigated. In addition, the association of common polymorphisms with PE predisposition and levels of the respective cytokines was never analysed in Chinese. We have genotyped common variants of IL17A, IL-23 and IL-12B and serum cytokine levels in Chinese PE patients and controls in the present study. Current reports shows that IL-17A (rs2275913) polymorphisms are associated with higher serum IL-17A levels and a predisposition to PE.

We observed elevated IL-17A levels in PE patients in comparison to the pregnant ladies and healthy cases. However, earlier reports remained controversial concerning the importance of IL-17A in PE. For example, a study by Jonsson et al. [24] showed no evidence of possible links between IL-17A levels and PE pathologic conditions. On the contrary, our result is corroborated with earlier observations indicating a remarkably higher titer of IL-17A in the sera of pregnant subjects complicated by fetal growth restriction (FGR) and PE as compared to healthy pregnant normotensive women [25]. These results were further strengthened by several other reports mentioning that there was a higher prevalence of Th17 cells in peripheral blood and enhanced expression of RORyt mRNA (transcription factor of Th17 cells) in placentas of pre-eclamptic subjects as compared normal healthy ones [12, 26, 27]. Gaestational period could also be a possible reason for the differential IL-17A levels between PE patients and healthy pregnant women.
Earlier investigations in the experimental model have deciphered the essential role of IL-17 in PE pathogenesis. Infusion of IL-17 to normal pregnant rats increased mean arterial pressure, elevated oxidative stress and enhanced Th17 cells [28]. Administration of Rituximab and superoxide dismutase to IL-17 infused rats improved pathogenesis by lowering the number of Th17 cells [28]. In addition, the administration of soluble IL-17 receptor significantly decreased Th17 cells, lowered blood pressure, and improved pathophysiological status of infused IL17 rats [29].

In contrast, we did not observe a possible difference in serum level IL-23 levels among three different studied groups. These results are contradictory to an earlier report [30] where a significantly lower level of this cytokine was demonstrated in pregnant groups (with and without PE) in comparison to healthy non-pregnant subjects. However, in line with our observations, a report by Darmochwal-Kolarz et al. [25] also failed to demonstrate the difference of IL-23 among pregnant subjects with placental insufficiency (fetal growth restriction and $\mathrm{PE}$ ) and healthy pregnant women. Our result showing a comparable level of IL-23 in subjects with PE and healthy pregnant women matched with the report, as mentioned above. Moreover, a comparable level of this cytokine in both the pregnant groups and healthy non-pregnant subjects indicate a negligible role of IL-23 in the context of pregnancy and its related complication in PE.

Common polymorphisms in the IL-17A gene have been associated with hypertension [31] and various organ dysfunctions [32]. As the primary clinical characteristics of $\mathrm{PE}$ are high blood pressure and dysfunction 
of kidney and liver, we hypothesized that variants in the IL-17A gene would be associated with predisposition to the development of PE. Out of three SNPs investigated in the present study, we observed a significant association of rs2275913 polymorphism with a predisposition to PE: heterozygous and minor allele was more frequent in PE cased when compared to pregnant women and healthy women. In contrast, the previous reports in Brazilian [33], Han Chinese [18], and the Iranian population [17] failed to demonstrate such association. Similarly, other variants of IL-17A polymorphism (rs1974226 and rs374806) were also not associated with susceptibility to PE. Furthermore, IL-23A (rs11171806) variants were also more frequent in $\mathrm{PE}$ compared to healthy and pregnant women indicating a susceptible genetic factor for PE development. The possible mechanism of how IL-23A rs11171806 is associated with PE susceptibility is not known. Mutation at 703 nucleic acids $(\mathrm{G}>\mathrm{A}$ ) position leads to a synonymous Ser106Ser, not affecting the three-dimensional structure of the IL-23 protein. Furthermore, in the present study, we also failed to observed differential IL-23 serum levels among different clinical categories. Also, the distribution of IL-12B (rs3212227) variants was comparable in $\mathrm{PE}$ patients and other clinical categories, indicating no significant role of IL-12B polymorphism in predisposition to PE development.

In the current report, a strong association of IL17A (rs2275913) and IL-23A (rs11171806) polymorphism with susceptibility to PE was observed. Also, we noticed elevated IL-17A levels in PE patients in comparison to healthy women and pregnant women, and IL-23 remained comparable. Based on these results, we hypothesized that common polymorphisms in IL-17A and IL-23A would be correlated with serum levels of IL-17A and IL-23, respectively. Serum levels of IL-23 were not associated with different genotypes of IL-23A gene (rs11171806), as the mutation $(\mathrm{G}>\mathrm{A})$ lead to no change in amino acids (Ser106Ser). Interestingly, IL-17A (rs2275913) polymorphism was observed to contributing serum levels of IL-17A: homozygous (AA) and heterozygous mutant (GA) displayed higher serum IL-17A compared to GG genotype. In line with the present report, earlier studies [31,34] have also demonstrated the functional relevance of IL-17A (rs2275913) with plasma or serum levels of IL-17A. Genetic variation at the promoter region of IL-17A gene would possibly enhance the binding of transcription factor and increased production of IL-17A cytokine.

\section{Conclusions}

The current report revealed an important role of IL$17 \mathrm{~A}$ in the pathogenesis of PE in Chinese patients. Furthermore, heterozygous mutant and minor allele of IL-17A (rs2275913) and IL-23A (rs11171806) polymorphisms predisposed subjects for the development of PE. Interestingly, the current report further re-validated the functional relevance of IL-17A (rs2275913) variants and demonstrated the association of mutants with elevated IL-17A levels. However, further studies, including more significant sample-sized in the different populations, are required to validate the observations of the present study.

\section{Abbreviations}

ELISA: Enzyme-linked immunosorbent assay; IL: Interlukin.

\section{Acknowledgements}

Authors would like to thanks all participants for the voluntary contribution for the present investigation.

\section{Authors' contributions}

$X L$ and $W L$ : performed experiments and prepare the first draft the manuscript; YH, WZ, XY, LC and QYinvolved in data analysis and interpretation; WC: made a contribution in the design, data interpretation, work supervision and critically revising the manuscript. All authors read and approved the manuscript.

\section{Funding}

This study was supported by The Interdisciplinary Program of Shanghai Jiao Tong University (No. ZH2018QNB16, No. ZH2018ZDA31), Tradational Chinese and Western medicine in Shanghai (No. 1841 1963500), and Important disease of Xuhui District Health Planning Commission (No. XHLHGG201805). The funders has no role in reporting of the manuscript.

\section{Availability of data and materials}

The datasets used and/or analysed during the current study are available from the corresponding author on reasonable request.

\section{Ethics approval and consent to participate}

The study was approved by the Clinical Research Ethics Committee of the Shanghai Jiao Tong University. Written informed consent was obtained from participants.

\section{Consent for publication}

Not applicable.

\section{Competing interests \\ Authors declear no conflict of interest.}

\section{Author details}

${ }^{1}$ The International Peace Maternity and Child Health Hospital, School of Medicine, Shanghai Jiao Tong University, 910 Hengshan Road, Shanghai 200030, China. ${ }^{2}$ Shanghai Municipal Key Clinical Specialty, Shanghai 200030, China. ${ }^{3}$ Department of Obstetrics and Gynecology, Shanghai Fourth People's Hospital, Tongji University, Shanghai 200030, China. ${ }^{4}$ Shanghai Key Laboratory of Embryo Original Diseases, Shanghai 200030, China.

Received: 18 November 2020 Accepted: 29 November 2020 Published online: 06 January 2021

\section{References}

1. Tjoa ML, Levine RJ, Karumanchi SA. Angiogenic factors and preeclampsia. Front Biosci. 2007;12:2395-402. 
2. Schindler AE. New data about preeclampsia: some possibilities of prevention. Gynecol Endocrinol. 2018;34:636-7.

3. Kuklina EV, Ayala C, Callaghan WM. Hypertensive disorders and severe obstetric morbidity in the United States. Obstet Gynecol. 2009:113:1299-306

4. Xiao J, Shen F, Xue Q, Chen G, Zeng K, Stone P, Zhao M, Chen Q. Is ethnicity a risk factor for developing preeclampsia? An analysis of the prevalence of preeclampsia in China. J Hum Hypertens. 2014;28:694-8.

5. Bowen JM, Chamley L, Mitchell MD, Keelan JA. Cytokines of the placenta and extra-placental membranes: biosynthesis, secretion and roles in establishment of pregnancy in women. Placenta. 2002;23:239-56.

6. Sargent IL, Borzychowski AM, Redman CW. Immunoregulation in normal pregnancy and pre-eclampsia: an overview. Reprod Biomed Online. 2006;13:680-6.

7. Raghupathy R, Makhseed M, Azizieh F, Omu A, Gupta M, Farhat R. Cytokine production by maternal lymphocytes during normal human pregnancy and in unexplained recurrent spontaneous abortion. Hum Reprod. 2000;15:713-8.

8. Raghupathy R, Al-Azemi M, Azizieh F. Intrauterine growth restriction: cytokine profiles of trophoblast antigen-stimulated maternal lymphocytes. Clin Dev Immunol. 2012;2012:734865.

9. Raghupathy R, Makhseed M, El-Shazly S, Azizieh F, Farhat R, Ashkanani L. Cytokine patterns in maternal blood after premature rupture of membranes. Obstet Gynecol. 2001;98:122-6.

10. Sykes L, Maclntyre DA, Yap XJ, Teoh TG, Bennett PR. The Th1:th2 dichotomy of pregnancy and preterm labour. Mediators Inflamm. 2012;2012:967629.

11. Benghiat FS, Charbonnier LM, Vokaer B, De Wilde V, Le Moine A. Interleukin 17-producing T helper cells in alloimmunity. Transplant Rev (Orlando). 2009;23:11-8.

12. Darmochwal-Kolarz D, Kludka-Sternik M, Tabarkiewicz J, Kolarz B, Rolinski J, Leszczynska-Gorzelak B, Oleszczuk J. The predominance of Th17 lymphocytes and decreased number and function of Treg cells in preeclampsia. J Reprod Immunol. 2012;93(2):75-81.

13. Ito M, Nakashima A, Hidaka T, Okabe M, Bac ND, Ina S, Yoneda S, Shiozaki A, Sumi S, Tsuneyama K, Nikaido T, Saito S. A role for IL-17 in induction of an inflammation at the fetomaternal interface in preterm labour. J Reprod Immunol. 2010;84:75-85.

14. Nakashima A, Ito M, Shima T, Bac ND, Hidaka T, Saito S. Accumulation of IL-17-positive cells in decidua of inevitable abortion cases. Am J Reprod Immunol. 2010;64:4-11.

15. Aggarwal S, Ghilardi N, Xie MH, de Sauvage FJ, Gurney AL. Interleukin-23 promotes a distinct CD4T cell activation state characterized by the production of interleukin-17. J Biol Chem. 2003;278:1910-4.

16. Darmochwal-Kolarz D, Kludka-Sternik M, Tabarkiewicz J, Kolarz B, Rolinski J, Leszczynska-Gorzelak B, Oleszczuk J. The predominance of Th17 lymphocytes anddecreased number and function of Treg cells in preeclampsia. J Reprod Immunol. 2012;93(2):75-81.

17. Anvari F, Dabagh-Gorjani F, Soltani-Zangbar MS, Kamali-Sarvestani E, Malek-Hosseini Z, Gharesi-Fard B. Investigating the association of IL-17A and IL-17F with susceptibility to pre-eclampsia in Iranian women. Iran J Immunol. 2015;12(2):117-28.

18. Wang H, Guo M, Liu F, Wang J, Zhou Z, Ji J, Ye Y, Song W, Liu S, Sun B, Role of IL-17 variants in preeclampsia in Chinese Han women. PLoS One. 2015;10(10):e0140118.

19. Wang X, Guo M, Li S, Gong J, Song W, Wang H, Liu S. The role of the IL-12 polymorphism rs3212227 in preeclampsia in Chinese Han women. Clin Exp Hypertens (New York, NY: 1993). 2016;38(4):388-92.
20. Bulletins--Obstetrics ACoP. ACOG practice bulletin. Diagnosis and management of preeclampsia and eclampsia. Number 33, January 2002. Obstet Gynecol. 2002;99(1):159-67.

21. Chen X, Han S, Wang S, Zhou X, Zhang M, Dong J, Shi X, Qian N, Wang X, Wei $Q$, et al. Interactions of IL-12A and IL-12B polymorphisms on the risk of cervical cancer in Chinese women. Clin Cancer Res. 2009;15(1):400-5.

22. Keramat F, Kazemi S, Saidijam M, Zamani A, Kohan HF, Mamani M, Eini P, Moghimbigi A, Alikhani MY. Association of interleukin-17 gene polymorphisms and susceptibility to brucellosis in Hamadan, western Iran. Microbiol Immunol. 2019;63(3-4):139-46.

23. Jia H, Tao F, Liu C, Guo T, Zhu W, Wang S, Cui B, Ning G. Both interleukin$23 \mathrm{~A}$ polymorphism and serum interlukin-23 expression are associated with Graves' disease risk. Cell Immunol. 2015;294(1):39-43.

24. Jonsson Y, Ruber M, Matthiesen L, Berg G, Nieminen K, Sharma S, Ernerudh J, Ekerfelt C. Cytokine mapping of sera from women with preeclampsia and normal pregnancies. J Reprod Immunol. 2006;70(1-2):83-91.

25. Darmochwal-Kolarz D, Michalak M, Kolarz B, Przegalinska-Kalamucka M, Bojarska-Junak A, Sliwa D, Oleszczuk J. The role of Interleukin-17, Interleukin-23, and transforming growth factor-beta in pregnancy complicated by placental insufficiency. Biomed Res Int. 2017;2017:6904325.

26. Toldi G, Rigo J Jr, Stenczer B, Vasarhelyi B, Molvarec A. Increased prevalence of IL-17-producing peripheral blood lymphocytes in pre-eclampsia. Am J Reprod Immunol. 2011;66(3):223-9.

27. Jianjun Z, Yali H, Zhiqun W, Mingming Z, Xia Z. Imbalance of T-cell transcription factors contributes to the Th1 type immunity predominant in pre-eclampsia. Am J Reprod Immunol. 2010;63(1):38-45.

28. Dhillion P, Wallace K, Herse F, Scott J, Wallukat G, Heath J, Mosely J, Martin JN Jr, Dechend R, LaMarca B. IL-17-mediated oxidative stress is an important stimulator of AT1-AA and hypertension during pregnancy. Am J Phys Regul Integr Comp Phys. 2012;303(4):R353-8.

29. Cornelius DC, Hogg JP, Scott J, Wallace K, Herse F, Moseley J, Wallukat G, Dechend R, LaMarca B. Administration of interleukin-17 soluble receptor C suppresses TH17 cells, oxidative stress, and hypertension in response to placental ischemia during pregnancy. Hypertension. 2013;62(6):1068-73.

30. Poordast T, Najib FS, Baharlou R, Bijani A, Alamdarloo SM, Poordast A. Assessment of Thelper 17-associated cytokines in third trimester of pregnancy. Iran J Immunol. 2017;14(2):172-9.

31. Huang H-T, Lu Y-L, Wang R, Qin H-M, Wang C-F, Wang J-L, Xiang Y, GUo J, Lan Y, Wei Y-S. The association of IL-17A polymorphisms with IL-17A serum levels and risk of ischemic stroke. Oncotarget. 2017:8(61):103499-508.

32. Domanski L, Kłoda K, Patrzyk M, Wisniewska M, Safranow K, Sienko J, Sulikowski T, Staniszewska M, Pawlik A. IL17A and IL17F genes polymorphisms are associated with histopathological changes in transplanted kidney. BMC Nephrol. 2019;20(1):124.

33. Tanaka SCSV, Hortolani ACC, Pissetti CW, Paschoini MC, Cintra-Ruiz MT, VR J, Balarin MAS. Polymorphisms in the IL17A gene are not involved in the development of preeclampsia in the Brazilian population. J Bras Patol Med Lab. 2019;55(2):170-81.

34. Tang H, Pei H, Xia Q, Tang Y, Huang J, Huang J, Pei F. Role of gene polymorphisms/haplotypes and serum levels of interleukin-17A in susceptibility to viral myocarditis. Exp Mol Pathol. 2018;104(2):140-5.

\section{Publisher's Note}

Springer Nature remains neutral with regard to jurisdictional claims in published maps and institutional affiliations. 